\title{
BAIT FORMULATIONS OF MOLLUSCICIDES AND THEIR EFFECTS ON BIOCHEMICAL CHANGES IN THE OVOTESTIS OF SNAIL Lymnaea acuminata (MOLLUSCA; GASTROPODA:LYMNAEIDAE)
}

Pradeep KUMAR, Vinay Kumar SINGH \& D.K. SINGH

\begin{abstract}
SUMMARY
The effect of sub-lethal feeding of bait formulations containing molluscicidal component of Ferula asafoetida (ferulic acid, umbelliferone), Syzygium aromaticum (eugenol) and Carum carvi (limonene) on biochemical changes in the ovotestis of snail Lymnaea acuminata were studied. Bait formulations feeding to L. acuminata were studied in clear glass aquaria having diameter of $30 \mathrm{~cm}$. Baits were prepared from different binary combinations of attractant amino acid (valine, aspartic acid, lysine and alanine $10 \mathrm{mM}$ ) in $100 \mathrm{~mL}$ of $2 \%$ agar solution + sub-lethal $\left(20 \%\right.$ and $60 \%$ of $\left.24 \mathrm{~h} \mathrm{LC}_{50}\right)$ doses of different molluscicides (ferulic acid, umbelliferone, eugenol and limonene). These baits caused maximum significant reduction in free amino acid, protein, DNA, RNA levels i.e. 41.37, 23.56, 48.36 and $14.29 \%$ of control in the ovotestis of the snail, respectively. Discontinuation of feeding after treatment of $60 \%$ of $96 \mathrm{LC}_{50}$ of molluscicide containing bait for next $72 \mathrm{~h}$ caused a significant recovery in free amino acid, protein, DNA and RNA levels in the ovotestis of L. acuminata.
\end{abstract}

KEYWORDS: Bait formulation; Molluscicides; Amino acids; Lymnaea acuminata; Biochemical changes.

\section{INTRODUCTION}

Fasciola hepatica and F. gigantica are the causative agent of endemic fascioliasis in different part of world ${ }^{14}$. This disease belongs to the plantborne trematods zoonoses. The definite host is very broad and includes many herbivorous mammals, including humans. Bovine fascioliasis is very common in the eastern region of Uttar Pradesh, India ${ }^{19}$. One way to reduce the incidence of fascioliasis is to de-link the life cycle of fluke, by destroying the intermediate host snails $\mathrm{s}^{4,7,8,10}$. The use of a combination of a feeding attractant and toxicant in the bait formulation is a good tool for pest management and has toxicological and ecological advantages over the release of molluscicides directly in the water ${ }^{1,9,16,24}$. It is therefore important to identify strong attractant and effective molluscicides for preparing bait formulations. Snails, like other gastropod molluscs, use chemical clues to locate food sources ${ }^{2,3,6,17,25,26,27}$. The freshwater snails inhabit an environment containing macrophytes algae and bacteria ${ }^{23}$. These aquatic organisms release different types of chemicals, such as carbohydrates and amino acids, into the surrounding water ${ }^{5,12,22,23}$ which acts as attractant for snails. The aim of the present study is to evaluate the effect of sub-lethal feeding molluscicides (ferulic acid, umbelliferone, eugenol and limonene) in bait formulations with attractant amino acid (valine, aspartic acid, lysine and alanine) on different biochemical changes (free amino acid, protein and nucleic acid) in the ovotestis of Lymnaea acuminata, a known vector of fascioliasis. Withdrawal experiments were also performed to study the reversibility of the effect on the snails.

\section{MATERIALS AND METHODS}

Test animals: The adult snails ( $2.25 \pm 0.20 \mathrm{~cm}$ in length) snails were collected locally from lakes and low lying submerged fields in Gorakhpur State of Uttar Pradesh in India. The snails were acclimatized for 72 hours in dechlorinated tap water at $25 \pm 1{ }^{\circ} \mathrm{C}$. The $\mathrm{pH}$ of the water was 7.2-7.3 and dissolved oxygen, free carbon dioxide and bicarbonate alkalinity were 6.5-7.3 mg/L, 5.2-6.3 mg/L and 102.0-106.0 mg/L, respectively.

Pure compounds: Agar-agar, amino acids (valine, aspartic acid, lysine and alanine), different active component (Molluscicides: eugenol, ferulic acid, umbelliferone and limonene) were used in bait formulation. The pure active component ferulic acid (4-Hydroxy-3 methoxycinnamic), umbelliferone (7-Hydroxy coumarin; 7-hydroxy-2H-1-benzopyran-2one), eugenol (2-Methoxy-4-(2-propenyl) phenol) and limonene ((R)4-Isopropenyl-1-methyl-1-cyclohexene): were purchased from Sigma chemical Co. (USA).

Preparation of bait formulations with molluscicides: Bait formulations containing binary combination of different amino acids (valine, aspartic acid, lysine and alanine $10 \mathrm{mM})$ and sub-lethal $(20 \%$ and $60 \%$ of $24 \mathrm{~h}$ and $96 \mathrm{hC}_{50}$ ) molluscicides were prepared in $100 \mathrm{~mL}$ of $2 \%$ agar solution by the method of $\mathrm{MADSEN}^{13}$. Concentrations of amino acids were based on the earlier reports of TIWARI \& SINGH ${ }^{25,26}$. These solutions were spread at a uniform thickness of $5 \mathrm{~mm}$. After 


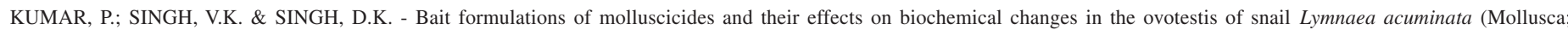
Gastropoda:Lymnaeidae). Rev. Inst. Med. Trop. Sao Paulo, 53(5): 271-5, 2011.

cooling, the bait containing sub-lethal molluscicides were cut out with a corer measuring $5 \mathrm{~mm}$ in diameter. Six replicates were prepared for each concentration. Control aquaria were left untreated. After $24 \mathrm{~h}$ of bait feeding the snails were washed with water and the ovotestis was removed from the snail and used for the measurement of biochemical changes. Different biochemical changes viz. free amino acid, protein, DNA and RNA were measured by feeding snails as well as a control group of snails.

In a withdrawal experiment free amino acid, protein, DNA and RNA level in the ovotestis of snail were measured in withdrawn snails after $96 \mathrm{~h}$ feeding to $60 \%$ of $96 \mathrm{hC}_{50}$ of bait feeding for next $72 \mathrm{~h}$ to fresh water.

Assay apparatus and procedure: The bioassay was performed by the method by TIWARI \& SINGH ${ }^{25,26}$. The bioassay chamber consists of a clean glass aquarium having a diameter of $30 \mathrm{~cm}$. Each aquarium was divided into four concentric zones with diameters of 13, 18, 24 and 30 $\mathrm{cm}$ : Central zone (zone 3), Middle zone (zone 2 and 1) and Outer zone (zone 0). A small annular elevation of $9 \mathrm{~mm}$ height and $2.4 \mathrm{~cm}$ diameter was made in the centre of aquarium (Zone 3). Zone 0 had an area of 254 $\mathrm{cm}^{2}$ on the periphery of aquarium. The aquaria were then filled with 500 $\mathrm{mL}$ of dechlorinated tap water to a height of $8 \mathrm{~mm}$ and maintained at $25 \pm 1{ }^{\circ} \mathrm{C}$. At the start of the assay ten individually marked snails were placed on the circumference of zone 0 . The distance between two snails was $66 \mathrm{~mm}$. Simultaneously, one of the prepared bait of different active component (molluscicides) was added on the small annular elevation in the center (Zone 3). Six sets of experiments have been designed with ten snails in each replicate. Snails were fed with sub-lethal i.e. $20 \%$ and $60 \%$ of $24 \mathrm{~h} \mathrm{LC}_{50}$ and $96 \mathrm{~h} \mathrm{LC}_{50}$ of the ferulic acid, umbelliferone, eugenol and limonene containing bait formulations. After $24 \mathrm{~h} / 96 \mathrm{~h}$ of feeding changes in the levels of protein, the total free amino acid, nucleic acid (DNA/RNA) in ovotestis of snails was measured. These changes were also studied in ovotestis of L. acuminata withdrawn from $96 \mathrm{~h}$ feeding for next $72 \mathrm{~h}$.

\section{Biochemical estimations}

Estimation of protein and free amino acids: Protein estimations $(\mu \mathrm{g} / \mathrm{mg})$ were made according to the method of LOWRY et al. ${ }^{11}$ using bovine serum albumin as a standard. Ten percent trichloroacetic acid (TCA: w/v) was used to prepare homogenates of tissue. Total free amino $\operatorname{acid}(\mu \mathrm{g} / \mathrm{mg})$ estimations were made according to the method of SPICE ${ }^{21}$.

Nucleic Acids: Estimation of DNA and RNA $(\mu \mathrm{g} / \mathrm{mg})$ were prepared by the method of SCHNEIDER ${ }^{15}$ using diphenylamine and orcinol. Homogenates $(1 \mathrm{mg} / \mathrm{mL}$,w/v) of ovotestis were prepared in $10 \%$ TCA at $90{ }^{\circ} \mathrm{C}$ and centrifuged at $5000 \mathrm{~g}$. Supernatants were used for the DNA and RNA estimations.

Statistical analysis: Each result was six times replicate estimation (measurement in six different pools of ovotestis). The values were expressed as mean \pm SE. Student's t-test was applied to determine the significant $(p<0.05)$ difference between treated and control animals ${ }^{20}$.

\section{RESULTS}

There was a significant $(p<0.05)$ decrease in protein levels in the ovotestis of snail L. acuminata fed to $20 \%$ and $60 \%$ of $24 \mathrm{~h}$ and $96 \mathrm{LC}_{50}$ of ferulic acid, umbelliferone, eugenol and limonene (Table 1). Maximum reduction $(23.56 \mu \mathrm{g} / \mathrm{mg}$ of control) in protein levels was observed in the ovotestis of L. acuminata fed to $60 \%$ of $96 \mathrm{~h} \mathrm{LC}_{50}$ of eugenol (Table $1)$. Significant $(p<0.05)$ recovery in protein level was observed in the ovotestis of L. acuminata $96 \mathrm{~h}$, when feeding was discontinued for the next $72 \mathrm{~h}$.

Sub-lethal feeding to $20 \%$ and $60 \%$ of $24 \mathrm{~h} \mathrm{LC}_{50}$ and $96 \mathrm{~h} \mathrm{LC}_{50}$ of eugenol, ferulic acid, umbelliferone, and limonene caused a significant decrease in the total free amino acid levels in the ovotestis of the snail $L$. acuminata (Table 2). Maximum decrease (41.37\% of control) in the total free amino acid was observed in the ovotestis of the snails fed to $60 \%$ of $96 \mathrm{~h} \mathrm{LC}_{50}$ of ferulic acid (Table 2). There was a significant $(p<0.05)$ recovery in the amino acid level in the ovotestis of withdrawn snails.

Significant decrease $(p<0.05)$ in DNA and RNA levels were observed in the ovotestis of L. acuminata fed to $20 \%$ and $60 \%$ of $24 \mathrm{~h} \mathrm{LC}_{50}$ and 96h $\mathrm{LC}_{50}$ of eugenol, ferulic acid, umbelliferone, and limonene (Tables $3,4)$. Maximum reduction in RNA ( $14.29 \%$ of control) and DNA $(48.36 \%$ of control) levels were observed in the ovotestis of the snail exposed to $60 \%$ of $96 \mathrm{~h} \mathrm{LC}_{50}$ of limonene and umbelliferone, respectively (Tables $3,4)$. Significant $(p<0.05)$ recovery in RNA, DNA levels were observed in the ovotestis of withdrawn snails.

\section{DISCUSSION}

It is evident from the results section that active molluscicidal components of Ferula asafoetida (ferulic acid, umbelliferone), Syzygium aromaticum (eugenol) and Carum carvi (limonene) in bait formulations were more effective in killing the L. acuminata. Earlier, it had been reported that direct release of ferulic acid, umbelliferone, eugenol and limonene in aquarium water have significant molluscicidal activity against $L$. acuminata $a^{7,10}$. The present study clearly demonstrates that when these active molluscicidal components in bait formulations were fed to snails, it also acts as potent molluscicides. Mode of entry of molluscicide into the snail's body is through the digestive system as it was used as bait. In an earlier study it was through the body surface when molluscicides were released directly in water. Although the entry of molluscicide inside the body is different, both methods are equally effective in killing the snails. Snails fed with a sub-lethal dose i.e. $20 \%$ and $60 \%$ of $24 \mathrm{~h}$ and $96 \mathrm{hC}_{50}$ of different molluscicides inside snail attractant pellets, caused a significant change in free amino acid, protein, nucleic acid (DNA and RNA) in the ovotestis of snail L. acuminata. The reduction in protein levels may be due to the direct interference of the active molluscicidal component. KUMAR et al. ${ }^{10}$ reported that there was a depletion of amino acids and reduction of protein and nucleic acid level in the ovotestis of L. acuminata when directly released in the aquarium. Due to depletion of free amino acids, there is a significant decrease in the levels of protein. The reduction in levels of proteins in the ovotestis of the treated snail may be due to the reduction synthesis of RNA, along with DNA ${ }^{10,18}$.

It can be concluded from the above study the reduction of free amino acid, protein and nucleic acid in the ovotestis of snail L. acuminata fed to bait containing active molluscicidal component could control the reproductive capacity of the snails even at sublethal doses. An added advantage of using the plant derived active components in baits is demonstrated by significant recovery in biochemical parameters in ovotestis of snails after discontinuation of feeding. It indicates that if there will be any environmental toxicity, it would be short term. 


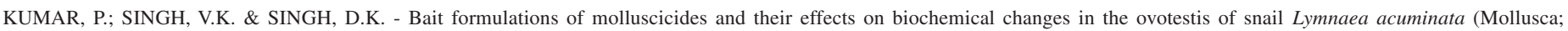
Gastropoda:Lymnaeidae). Rev. Inst. Med. Trop. Sao Paulo, 53(5): 271-5, 2011.

Table 1

Effect of sublethal exposure ( $20 \%$ and $60 \%$ of $24 \mathrm{~h} \mathrm{LC}_{50}$ and $96 \mathrm{~h} \mathrm{LC}_{50}$ ) of bait formulations with active molluscicidal component (eugenol, ferulic acid, umbelliferone, and limonene) on the level of proteins $(\mu \mathrm{g} / \mathrm{mg})$ in the ovotestis of the snail L. acuminata

\begin{tabular}{|c|c|c|c|c|c|}
\hline \multirow[t]{2}{*}{ Treatment } & \multicolumn{2}{|c|}{$24 \mathrm{~h} \mathrm{LC}_{50}$} & \multicolumn{2}{|c|}{$96 \mathrm{~h} \mathrm{LC}_{50}$} & \multirow{2}{*}{$\begin{array}{c}\text { Withdrawal } \\
60 \%\left(96 \mathrm{LC}_{50}\right)\end{array}$} \\
\hline & $20 \%$ & $60 \%$ & $20 \%$ & $60 \%$ & \\
\hline Vali+Aspa+Eug & $23.62 \pm 0.65 *(25.19)$ & $22.85 \pm 0.70 *(24.37)$ & $23.11 \pm 0.96 *(24.64)$ & $22.85 \pm 0.56 *(24.37)$ & $23.85 \pm 0.96+(24.21)$ \\
\hline Vali+Aspa+Fer & $38.62 \pm 0.36 *(41.19)$ & $35.96 \pm 0.81 *(38.35)$ & $37.56 \pm 0.36 *(40.05)$ & $33.96 \pm 0.81 *(36.22)$ & $38.92 \pm 0.38+(41.14)$ \\
\hline Vali+Aspa+Umb & $26.96 \pm 0.31 *(28.75)$ & $25.75 \pm 0.85 *(27.46)$ & $26.66 \pm 0.57 *(28.43)$ & $24.96 \pm 0.86 *(26.62)$ & $26.96 \pm 0.55+(28.49)$ \\
\hline Vali+Aspa+Lim & $33.96 \pm 0.77 *(36.22)$ & $31.80 \pm 0.13 *(33.91)$ & $30.65 \pm 0.30 *(32.68)$ & $29.08 \pm 0.17 *(31.01)$ & $32.75 \pm 0.66+(34.61)$ \\
\hline Lysi+Vali+Eug & $24.21 \pm 0.70 *(25.52)$ & $23.96 \pm 0.80 *(25.26)$ & $24.11 \pm 0.18 *(25.41)$ & $23.06 \pm 0.88 *(24.33)$ & $25.96 \pm 0.46+(27.69)$ \\
\hline Lysi+Vali+Fer & $35.16 \pm 0.63 *(37.06)$ & $34.66 \pm 0.85 *(36.54)$ & $34.96 \pm 0.81 *(36.85)$ & $31.75 \pm 0.62 *(33.47)$ & $34.75 \pm 0.31+(37.06)$ \\
\hline Lysi+Vali+Umb & $26.72 \pm 0.80 *(28.17)$ & $25.10 \pm 0.55 *(26.46)$ & $25.02 \pm 0.19 *(26.37)$ & $24.03 \pm 0.17 *(25.33)$ & $26.11 \pm 0.72+(27.85)$ \\
\hline Lysi+Vali+Lim & $36.55 \pm 0.26 *(38.53)$ & $25.75 \pm 0.23 *(27.14)$ & $35.33 \pm 0.87 *(37.24)$ & $24.96 \pm 0.85 *(26.31)$ & $28.96 \pm 0.21+(30.89)$ \\
\hline Lysi+Ala+Eug & $24.62 \pm 0.76 *(26.54)$ & $23.11 \pm 0.96 *(24.91)$ & $23.85 \pm 0.36 *(25.71)$ & $21.86 \pm 0.62 *(23.56)$ & $23.98 \pm 0.12+(25.55)$ \\
\hline Lysi+Ala+Fer & $35.96 \pm 0.66 *(38.76)$ & $31.82 \pm 0.75 *(34.30)$ & $34.96 \pm 0.70 *(37.68)$ & $33.96 \pm 0.62 *(36.61)$ & $34.81 \pm 0.38+(37.09)$ \\
\hline Lysi+Ala+Umb & $25.63 \pm 0.26 *(27.63)$ & $24.98 \pm 0.88 *(26.92)$ & $25.11 \pm 0.73 *(27.06)$ & $23.75 \pm 0.66 *(25.60)$ & $25.03 \pm 0.82+(26.67)$ \\
\hline Lysi+Ala+Lim & $34.76 \pm 0.63 *(37.47)$ & $33.96 \pm 0.33 *(36.61)$ & $33.05 \pm 0.72 *(35.62)$ & $30.85 \pm 0.62 *(33.25)$ & $33.87 \pm 0.26+(36.08)$ \\
\hline Ala+Vali+Eug & $37.62 \pm 0.73 *(40.08)$ & $36.15 \pm 0.28 *(38.51)$ & $36.70 \pm 0.88 *(39.10)$ & $34.66 \pm 0.96 *(36.92)$ & $35.78 \pm 0.32+(38.16)$ \\
\hline Ala+Vali+Fer & $37.68 \pm 0.96 *(40.14)$ & $35.99 \pm 0.38 *(38.34)$ & $35.12 \pm 0.72 *(37.41)$ & $34.76 \pm 0.88 *(37.03)$ & $36.36 \pm 0.89+(38.78)$ \\
\hline Ala+Vali+Umb & $25.86 \pm 0.33 *(27.55)$ & $24.72 \pm 0.23 *(26.33)$ & $24.11 \pm 0.67 *(25.68)$ & $23.98 \pm 0.23 *(25.54)$ & $24.72 \pm 0.66+(26.36)$ \\
\hline Ala+Vali+Lim & $30.62 \pm 0.69 *(32.62)$ & $28.12 \pm 0.76 *(29.95)$ & $30.10 \pm 0.82 *(32.06)$ & $27.69 \pm 0.21 *(29.50)$ & $30.02 \pm 0.96+(23.02)$ \\
\hline Control (Agar) & \multicolumn{4}{|c|}{$94.63 \pm 0.63(100)$} & $90.62 \pm 0.73(100)$ \\
\hline Control (a) Vali+Aspa & \multicolumn{4}{|c|}{$93.76 \pm 0.82(100)$} & $94.60 \pm 0.81(100)$ \\
\hline Control (b) Lysi+Vali & \multicolumn{4}{|c|}{$94.85 \pm 0.68(100)$} & $93.15 \pm 0.63(100)$ \\
\hline Control (c) Lysi+Ala & \multicolumn{4}{|c|}{$93.76 \pm 0.88(100)$} & $93.85 \pm 0.62(100)$ \\
\hline Control (d) Ala+Vali & \multicolumn{4}{|c|}{$93.86 \pm 0.31(100)$} & $93.75 \pm 0.60(100)$ \\
\hline
\end{tabular}

Each value is mean \pm SE of six replicates. Value in parenthesis is per cent change with control taken as $100 \%$. Concentration (w/v) has been expressed as final concentration in aquarium water. $\left.{ }^{*}\right)$ Significant $(p<0.05)$ when ' $\mathrm{t}$ ' test was applied in between treated and control group and $(+)$ in between $60 \%$ of $96 \mathrm{~h} \mathrm{LC}_{50}$ and withdrawal group . Vali $=$ valine, Aspa $=$ aspartic acid, Lys $=$ lysine, Ala $=$ alanine, Eug $=$ eugenol, $\mathrm{Feb}=$ ferulic acid, Umb $=$ umbelliferone, Lim $=$ limonene .

Table 2

Effect of sublethal exposure ( $20 \%$ and $60 \%$ of $24 \mathrm{~h} \mathrm{LC}_{50}$ and $96 \mathrm{~h} \mathrm{LC}_{50}$ ) of bait formulations with active molluscicidal component (eugenol, ferulic acid, umbelliferone, and limonene) on the level of amino acid $(\mu \mathrm{g} / \mathrm{mg})$ in the ovotestis of the snail L. acuminata

\begin{tabular}{|c|c|c|c|c|c|}
\hline \multirow[t]{2}{*}{ Treatment } & \multicolumn{2}{|c|}{$24 \mathrm{~h} \mathrm{LC}_{50}$} & \multicolumn{2}{|c|}{$96 \mathrm{hCC}_{50}$} & \multirow{2}{*}{$\begin{array}{c}\text { Withdrawal } \\
60 \%\left(96 \mathrm{~h} \mathrm{LC} \mathrm{LC}_{50}\right)\end{array}$} \\
\hline & $20 \%$ & $60 \%$ & $20 \%$ & $60 \%$ & \\
\hline Vali+Aspa+Eug & $15.18 \pm 0.67 *(48.84)$ & $14.66 \pm 0.75^{*}(47.16)$ & $15.15 \pm 0.63 *(48.74)$ & $13.39 \pm 0.55^{*}(43.08)$ & $14.75 \pm 0.29+(47.45)$ \\
\hline Vali+Aspa+Fer & $14.68 \pm 0.32 *(47.23)$ & $14.12 \pm 0.13 *(45.43)$ & $13.99 \pm 0.25 *(45.01)$ & $12.86 \pm 0.36^{*}(41.37)$ & $13.75 \pm 0.55+(44.24)$ \\
\hline Vali+Aspa+Umb & $16.62 \pm 0.83 *(53.47)$ & $15.72 \pm 0.63 *(50.57)$ & $15.85 \pm 0.70 *(50.99)$ & $14.36 \pm 0.55 *(46.20)$ & $15.86 \pm 0.13+(51.02)$ \\
\hline Vali+Aspa+Lim & $15.50 \pm 0.96 *(49.87)$ & $14.99 \pm 0.83 *(48.23)$ & $15.63 \pm 0.96 *(50.28)$ & $14.85 \pm 0.63 *(47.77)$ & $16.11 \pm 0.85+51.83)$ \\
\hline Lysi+Vali+Eug & $16.66 \pm 0.75 *(53.48)$ & $15.32 \pm 0.32 *(49.18)$ & $16.38 \pm 0.31 *(52.58)$ & $15.96 \pm 0.81 *(51.23)$ & $16.89 \pm 0.36+(53.90)$ \\
\hline Lysi+Vali+Fer & $15.75 \pm 0.81 *(50.56)$ & $14.92 \pm 0.66 *(47.89)$ & $15.75 \pm 0.98 *(50.56)$ & $14.73 \pm 0.58 *(47.28)$ & $15.96 \pm 0.31+(56.94)$ \\
\hline Lysi+Vali+Umb & $16.82 \pm 0.55 *(53.99)$ & $15.93 \pm 0.72 *(51.13)$ & $15.90 \pm 0.45 *(510.4)$ & $14.82 \pm 0.48 *(47.57)$ & $16.31 \pm 0.82+(52.05)$ \\
\hline Lysi+Vali+Lim & $15.85 \pm 0.66 *(50.88)$ & $15.70 \pm 0.71 *(50.40)$ & $14.62 \pm 0.35 *(46.93)$ & $14.60 \pm 0.70 *(46.86)$ & $15.68 \pm 0.41+(50.04)$ \\
\hline Lysi+Ala+Eug & $16.66 \pm 0.40 *(53.43)$ & $15.99 \pm 0.85 *(51.28)$ & $16.82 \pm 0.82 *(53.94)$ & $15.71 \pm 0.38 *(50.38)$ & $16.92 \pm 0.80+(56.19)$ \\
\hline Lysi+Ala+Fer & $14.75 \pm 0.98 *(47.30)$ & $14.78 \pm 0.71 *(47.40)$ & $15.33 \pm 0.62 *(49.16)$ & $14.63 \pm 0.89 *(46.92)$ & $15.96 \pm 0.89+(53.00)$ \\
\hline Lysi+Ala+Umb & $16.66 \pm 0.75^{*}(53.43)$ & $15.96 \pm 0.38 *(51.18)$ & $16.30 \pm 0.77 *(52.27)$ & $15.96 \pm 0.80 *(51.18)$ & $16.76 \pm 0.32+(55.66)$ \\
\hline Lysi+Ala+Lim & $15.87 \pm 0.96 *(50.89)$ & $15.12 \pm 0.66^{*}(48.49)$ & $15.82 \pm 0.60 *(50.73)$ & $14.12 \pm 0.89 *(45.28)$ & $15.66 \pm 0.59+(52.00)$ \\
\hline Ala+Vali+Eug & $14.66 \pm 0.70 *(47.00)$ & $14.12 \pm 0.68 *(45.27)$ & $14.75 \pm 0.51 *(47.29)$ & $13.48 \pm 0.76^{*}(43.21)$ & $14.96 \pm 0.66+(49.58)$ \\
\hline $\mathrm{Ala}+\mathrm{Vali}+\mathrm{Fer}$ & $15.63 \pm 0.61 *(51.11)$ & $15.87 \pm 0.36 *(5088)$ & $15.11 \pm 0.42 *(48.44)$ & $14.96 \pm 0.82 *(47.96)$ & $15.86 \pm 0.21+(52.56)$ \\
\hline $\mathrm{Ala}+\mathrm{Vali}+\mathrm{Umb}$ & $15.76 \pm 0.96 *(50.52)$ & $14.85 \pm 0.96 *(47.61)$ & $15.60 \pm 0.36 *(50.01)$ & $13.33 \pm 0.82 *(42.72)$ & $15.87 \pm 0.85+(52.60)$ \\
\hline Ala+Vali+Lim & $14.62 \pm 0.36 *(46.87)$ & $15.96 \pm 0.81 *(51.17)$ & $15.07 \pm 0.87 *(48.31)$ & $14.88 \pm 0.62 *(47.70)$ & $16.82 \pm 0.36+(55.75)$ \\
\hline Control (Agar) & \multicolumn{4}{|c|}{$30.12 \pm 0.15(100)$} & $28.33 \pm 0.13(100)$ \\
\hline Control (a) Vali+Aspa & \multicolumn{4}{|c|}{$31.08 \pm 0.11(100)$} & $31.33 \pm 0.18(100)$ \\
\hline Control (b) Lysi+Vali & \multicolumn{4}{|c|}{$31.15 \pm 0.06(100)$} & $31.15 \pm 0.12(100)$ \\
\hline Control (c) Lysi+Ala & \multicolumn{4}{|c|}{$31.18 \pm 0.18(100)$} & $30.11 \pm 0.11(100)$ \\
\hline Control (d) Ala+Vali & \multicolumn{4}{|c|}{$31.19 \pm 0.66(100)$} & $30.17 \pm 0.07(100)$ \\
\hline
\end{tabular}

Each value is mean $\pm \mathrm{SE}$ of six replicates. Value in parenthesis is per cent change with control taken as $100 \%$. Concentration (w/v) has been expressed as final concentration in aquarium water. (*) Significant $(p<0.05)$ when ' $\mathrm{t}$ ' test was applied in between treated and control group and $(+)$ in between $60 \%$ of $96 \mathrm{~h} \mathrm{LC}_{50}$ and withdrawal group . Vali $=$ valine, Aspa $=$ aspartic acid, Lysi $=$ lysine, $\mathrm{Ala}=$ alanine, Eug $=$ eugenol, Feb $=$ ferulic acid, Umb $=$ umbelliferone, Lim $=$ limonene . 


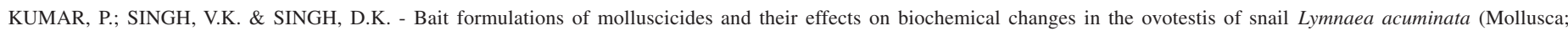
Gastropoda:Lymnaeidae). Rev. Inst. Med. Trop. Sao Paulo, 53(5): 271-5, 2011.

Table 3

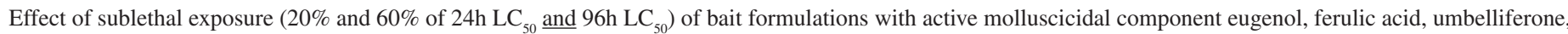
and limonene on the level of RNA $(\mu \mathrm{g} / \mathrm{mg})$ in the ovotestis of the snail L. acuminata

\begin{tabular}{|c|c|c|c|c|c|}
\hline \multirow[t]{2}{*}{ Treatment } & \multicolumn{2}{|c|}{$24 \mathrm{~h} \mathrm{LC}_{50}$} & \multicolumn{2}{|c|}{$96 \mathrm{hCC}_{50}$} & \multirow{2}{*}{$\begin{array}{c}\text { Withdrawal } \\
60 \%\left(96 h \mathrm{LC}_{50}\right)\end{array}$} \\
\hline & $20 \%$ & $60 \%$ & $20 \%$ & $60 \%$ & \\
\hline Vali+Aspa+Eug & $13.66 \pm 0.72 *(24.44)$ & $11.87 \pm 0.96 *(21.23)$ & $12.63 \pm 0.82 *(22.59)$ & $10.75 \pm 0.82 *(19.23)$ & $12.85 \pm 0.76+(23.42)$ \\
\hline Vali+Aspa+Fer & $10.75 \pm 0.22 *(19.23)$ & $9.85 \pm 0.65 *(17.62)$ & $9.99 \pm 0.83 *(17.87)$ & $8.96 \pm 0.33 *(16.03)$ & $10.12 \pm 0.82+(18.44)$ \\
\hline Vali+Aspa+Umb & $10.66 \pm 0.39 *(19.07)$ & $9.66 \pm 0.72 *(17.28)$ & $9.12 \pm 0.70 *(16.31)$ & $8.23 \pm 0.62 *(14.72)$ & $9.98 \pm 0.62+(18.19)$ \\
\hline Vali+Aspa+Lim & $11.82 \pm 0.76 *(21.14)$ & $9.88 \pm 0.32 *(17.67)$ & $10.87 \pm 0.14 *(19.44)$ & $9.66 \pm 0.92 *(17.28)$ & $10.79 \pm 0.28+(19.66)$ \\
\hline Lysi+Vali+Eug & $12.38 \pm 0.69 *(21.86)$ & $11.72 \pm 0.63 *(20.69)$ & $11.98 \pm 0.33 *(21.15)$ & $10.33 \pm 0.41 *(18.24)$ & $11.86 \pm 0.73+(20.90)$ \\
\hline Lysi+Vali+Fer & $11.61 \pm 0.77 *(20.50)$ & $10.63 \pm 0.71 *(18.77)$ & $10.60 \pm 0.34 *(18.71)$ & $9.86 \pm 0.72 *(17.09)$ & $10.99 \pm 0.89+(19.37)$ \\
\hline Lysi+Vali+Umb & $9.86 \pm 0.56 *(17.41)$ & $8.24 \pm 0.69 *(14.55)$ & $9.80 \pm 0.77 *(17.30)$ & $8.11 \pm 0.52 *(14.32)$ & $9.83 \pm 0.73+(17.33)$ \\
\hline Lysi+Vali+Lim & $10.26 \pm 0.11 *(18.11)$ & $9.66 \pm 0.32 *(17.05)$ & $10.12 \pm 0.69 *(17.87)$ & $9.10 \pm 0.94 *(16.06)$ & $10.70 \pm 0.98+(18.86)$ \\
\hline Lysi+Ala+Eug & $12.76 \pm 0.48 *(22.48)$ & $10.62 \pm 0.55 *(18.71)$ & $11.96 \pm 0.72 *(21.07)$ & $10.13 \pm 0.58 *(17.84)$ & $11.96 \pm 0.16+(22.90)$ \\
\hline Lysi+Ala+Fer & $11.19 \pm 0.38 *(19.71)$ & $10.62 \pm 0.52 *(18.71)$ & $10.72 \pm 0.96 *(18.88)$ & $9.66 \pm 0.92 *(17.01)$ & $10.82 \pm 0.72+(19.92)$ \\
\hline Lysi+Ala+Umb & $10.33 \pm 0.26 *(18.19)$ & $9.70 \pm 0.58 *(17.08)$ & $9.66 \pm 0.31 *(17.01)$ & $8.69 \pm 0.38 *(15.31)$ & $9.62 \pm 0.12+(17.71)$ \\
\hline Lysi+Ala+Lim & $10.99 \pm 0.55 *(19.36)$ & $9.86 \pm 0.39 *(17.37)$ & $9.82 \pm 0.33 *(17.30)$ & $8.83 \pm 0.72 *(15.55)$ & $9.26 \pm 0.73+(17.05)$ \\
\hline Ala+Vali+Eug & $12.92 \pm 0.37 *(22.75)$ & $10.24 \pm 0.71 *(18.03)$ & $11.62 \pm 0.11 *(20.46)$ & $9.67 \pm 0.38 *(17.02)$ & $10.75 \pm 0.23+(19.32)$ \\
\hline $\mathrm{Ala}+\mathrm{Vali}+\mathrm{Fer}$ & $11.32 \pm 0.82 *(19.93)$ & $10.31 \pm 0.23 *(18.15)$ & $10.11 \pm 0.73 *(17.80)$ & $9.22 \pm 0.83 *(16.23)$ & $10.96 \pm 0.76+(19.70)$ \\
\hline $\mathrm{Ala}+\mathrm{Vali}+\mathrm{Umb}$ & $10.12 \pm 0.32 *(17.82)$ & $8.13 \pm 0.81 *(16.07)$ & $9.66 \pm 0.51 *(17.01)$ & $8.36 \pm 0.82 *(14.72)$ & $9.62 \pm 0.82+(17.29)$ \\
\hline Ala+Vali+Lim & $10.11 \pm 0.62 *(17.80)$ & $9.08 \pm 0.66 *(15.98)$ & $9.08 \pm 0.67 *(15.98)$ & $8.12 \pm 0.89 *(14.29)$ & $9.65 \pm 0.23+(17.34)$ \\
\hline Control (Agar) & \multicolumn{4}{|c|}{$57.66 \pm 0.88(100)$} & $56.63 \pm 0.78(100)$ \\
\hline Control (a) Vali+Aspa & \multicolumn{4}{|c|}{$55.89 \pm 0.62(100)$} & $54.86 \pm 0.13(100)$ \\
\hline Control (b) Lysi+Vali & \multicolumn{4}{|c|}{$56.63 \pm 0.96(100)$} & $56.72 \pm 0.82(100)$ \\
\hline Control (c) Lysi+Ala & \multicolumn{4}{|c|}{$56.76 \pm 0.72(100)$} & $54.31 \pm 0.96(100)$ \\
\hline Control (d) Ala+Vali & \multicolumn{4}{|c|}{$56.79 \pm 0.92(100)$} & $55.62 \pm 0.85(100)$ \\
\hline
\end{tabular}

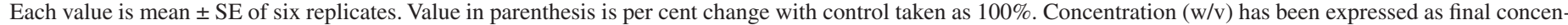

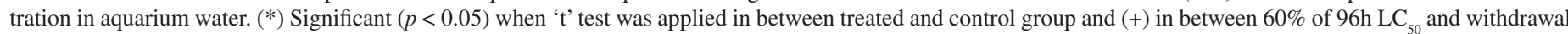
group. Vali $=$ valine, Aspa $=$ aspartic acid, Lysi $=$ lysine, Ala $=$ alanine, Eug $=$ eugenol, Feb $=$ ferulic acid, Umb $=$ umbelliferone, Lim $=$ limonene .

Table 4

Effect of sublethal exposure ( $20 \%$ and $60 \%$ of $24 \mathrm{~h} \mathrm{LC}_{50}$ and $96 \mathrm{~h} \mathrm{LC} \mathrm{L}_{50}$ ) of bait formulations with active molluscicidal component (eugenol, ferulic acid, umbelliferone, and limonene) on the level of DNA $(\mu \mathrm{g} / \mathrm{mg})$ in the ovotestis of the snail L. acuminata

\begin{tabular}{|c|c|c|c|c|c|}
\hline \multirow[t]{2}{*}{ Treatment } & \multicolumn{2}{|c|}{$24 \mathrm{~h} \mathrm{LC}_{50}$} & \multicolumn{2}{|c|}{$96 \mathrm{~h} \mathrm{LC}_{50}$} & \multirow{2}{*}{$\begin{array}{c}\text { Withdrawal } \\
60 \%\left(96 \mathrm{~h} \mathrm{LC}_{50}\right)\end{array}$} \\
\hline & $20 \%$ & $60 \%$ & $20 \%$ & $60 \%$ & \\
\hline Vali+Aspa+Fer & $33.96 \pm 0.42 *(55.38)$ & $32.11 \pm 0.62 *(52.36)$ & $33.82 \pm 0.56 *(55.18)$ & $30.31 \pm 0.98 *(49.42)$ & $32.98 \pm 0.26+(56.27)$ \\
\hline Vali+Aspa+Umb & $35.82 \pm 0.75 *(58.41)$ & $34.61 \pm 0.87 *(56.44)$ & $35.18 \pm 0.63 *(57.37)$ & $33.75 \pm 0.82 *(55.03)$ & $34.50 \pm 0.76+(58.87)$ \\
\hline Vali+Aspa+Lim & $35.96 \pm 0.38 *(60.27)$ & $34.72 \pm 0.72 *(56.62)$ & $34.85 \pm 0.76 *(56.83)$ & $33.98 \pm 0.51 *(55.41)$ & $35.96 \pm 0.32+(61.36)$ \\
\hline Lysi+Vali+Eug & $43.98 \pm 0.27 *(72.47)$ & $40.69 \pm 0.81 *(67.05)$ & $42.76 \pm 0.22 *(70.46)$ & $40.11 \pm 0.62 *(66.10)$ & $42.75 \pm 0.96+(75.05)$ \\
\hline Lysi+Vali+Fer & $31.76 \pm 0.96 *(52.34)$ & $30.22 \pm 0.70 *(49.80)$ & $30.66 \pm 0.50 *(50.52)$ & $29.86 \pm 0.63 *(49.20)$ & $31.10 \pm 0.62+(54.59)$ \\
\hline Lysi+Vali+Umb & $34.96 \pm 0.67 *(57.61)$ & $33.25 \pm 0.86 *(54.79)$ & $34.12 \pm 0.36 *(56.22)$ & $32.75 \pm 0.96 *(53.97)$ & $33.75 \pm 0.66+(59.25)$ \\
\hline Lysi+Vali+Lim & $36.69 \pm 0.72 *(60.46)$ & $34.88 \pm 0.79 *(57.48)$ & $36.10 \pm 0.27 *(59.49)$ & $34.50 \pm 0.69 *(56.85)$ & $35.99 \pm 0.61+(63.18)$ \\
\hline Lysi+Ala+Eug & $41.69 \pm 0.78 *(66.50)$ & $40.05 \pm 0.59 *(63.88)$ & $40.96 \pm 0.21 *(65.33)$ & $38.25 \pm 0.72 *(61.01)$ & $40.85 \pm 0.76+(65.92)$ \\
\hline Lysi+Ala+Fer & $33.75 \pm 0.39 *(53.83)$ & $31.85 \pm 0.66 *(50.80)$ & $33.12 \pm 0.78 *(52.83)$ & $30.62 \pm 0.38 *(48.84)$ & $32.67 \pm 0.68+(52.72)$ \\
\hline Lysi+Ala+Umb & $34.96 \pm 0.71 *(55.76)$ & $32.90 \pm 0.38 *(52.48)$ & $33.66 \pm 0.81 *(53.69)$ & $30,32 \pm 0.88 *(48.36)$ & $33.77 \pm 0.24+(54.50)$ \\
\hline Lysi+Ala+Lim & $35.68 \pm 0.55 *(57.20)$ & $33.62 \pm 0.72 *(53.62)$ & $32.98 \pm 0.89 *(52.60)$ & $30.79 \pm 0.96 *(49.11)$ & $34.24 \pm 0.82+(55.26)$ \\
\hline Ala+Vali+Eug & $42.85 \pm 0.25 *(70.50)$ & $41.66 \pm 0.78 *(68.54)$ & $40.62 \pm 0.56 *(66.83)$ & $38.75 \pm 0.39 *(63.75)$ & $40.85 \pm 0.96+(68.27)$ \\
\hline Ala+Vali+Fer & $32.72 \pm 0.62 *(53.83)$ & $30.66 \pm 0.96 *(50.44)$ & $31.85 \pm 0.14 *(52.40)$ & $29.72 \pm 0.39 *(48.89)$ & $31.96 \pm 0.44+(53.41)$ \\
\hline $\mathrm{Ala}+\mathrm{Vali}+\mathrm{Umb}$ & $35.62 \pm 0.72 *(58.60)$ & $33.88 \pm 0.79 *(55.74)$ & $33.79 \pm 0.85 *(55.59)$ & $30.62 \pm 0.72 *(50.37)$ & $32.85 \pm 0.62+(54.90)$ \\
\hline Ala+Vali+Lim & $35.86 \pm 0.82 *(58.99)$ & $33.87 \pm 0.62 *(55.72)$ & $34.85 \pm 0.77 *(57.33)$ & $33.28 \pm 0.11 *(54.75)$ & $34.66 \pm 0.73+(57.93)$ \\
\hline Control (Agar) & \multicolumn{4}{|c|}{$62.66 \pm 0.96(100)$} & $60.75 \pm 0.23(100)$ \\
\hline Control (a) Vali+Aspa & \multicolumn{4}{|c|}{$61.32 \pm 0.62(100)$} & $58.60 \pm 0.62(100)$ \\
\hline Control (b) Lysi+Vali & \multicolumn{4}{|c|}{$60.68 \pm 0.83(100)$} & $56.96 \pm 0.38(100)$ \\
\hline Control (c) Lysi+Ala & \multicolumn{4}{|c|}{$62.69 \pm 0.82(100)$} & $61.96 \pm 0.73(100)$ \\
\hline Control (d) Ala+Vali & \multicolumn{4}{|c|}{$60.78 \pm 0.98(100)$} & $59.83 \pm 0.86(100)$ \\
\hline
\end{tabular}

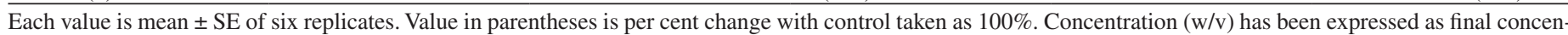

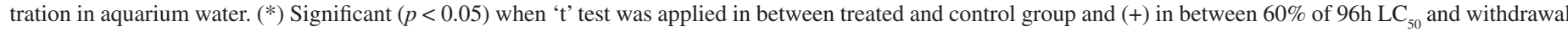
group. Vali $=$ valine, Aspa $=$ aspartic acid, Lysi $=$ lysine, Ala $=$ alanine, Eug $=$ eugenol, Feb $=$ ferulic acid, Umb $=$ umbelliferone, Lim $=$ limonene. 


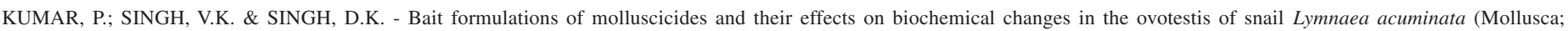
Gastropoda:Lymnaeidae). Rev. Inst. Med. Trop. Sao Paulo, 53(5): 271-5, 2011.

\section{ACKNOWLEDGMENTS}

One of the authors (Dr. Pradeep Kumar - Post Doctoral Fellow) is thankful to University Grants Commission, New Delhi, India for financial assistance.

\section{RESUMO}

\section{Formulações de iscas de moluscicidas e seus efeitos sobre as alterações bioquímicas no ovoteste do caramujo Lymnaea acuminata (Mollusca;Gastropoda:Lymnaeidae)}

Foi estudado o efeito subletal das iscas usadas para alimentação contendo componentes moluscicidas de Ferula asafoetida (ácido ferúlico, umbeliferone), Syzygium aromaticum (eugenol) e Carum carvi (limonene) nas alterações bioquímicas do ovoteste do caramujo Lymnaea acuminata. A formulação das iscas usadas para alimentar $L$. acuminata foi estudada em aquários de vidros transparentes de diâmetro de $30 \mathrm{~cm}$. As iscas foram preparadas por combinações diferentes binárias de aminoácidos (valina, ácido aspártico, lisina e alanina $10 \mathrm{mM}$ ) em $100 \mathrm{~mL}$ de solução de agar a $2 \%$ + doses subletais $(20 \%$ e $60 \%$ durante 24 horas $\mathrm{LC}_{50}$ ) de diferentes moluscicidas (ácido ferúlico, umbeliferone, eugenol e limonene). Estas iscas causaram redução significante máxima em aminoácidos livres, proteínas, níveis de DNA e RNA isto é 41,37\%, $23,56 \%, 48,36 \%$ e $14,29 \%$ de controle no ovoteste do caramujo, respectivamente. Discontinuação da alimentação depois do tratamento de $60 \%$ de 96 horas de $\mathrm{LC}_{50}$ do moluscicida contendo a isca para as subsequentes 72 horas causou significante recuperação dos níveis de aminoácidos livres, proteína, DNA e RNA no ovoteste da L. acuminata.

\section{REFERENCES}

1. Abdel-Hamid, AZ. Development of bait formulation for control of intermediate hosts of African Schistosome species. J Appl Toxicol. 1998;17:391-5.

2. Audesirk TE, Audesirk GJ. Behaviour of gastropod mollusks. In: Willow AOD, Wilbur KM, editors. The Mollusca, v. 8. London: Academic Press; 1985. p. 1-94.

3. Croll RP. Gastropod chemoreception. Biol Rev. 1983;58:293-319.

4. Godan D. Pest slugs and snails biology and control. Berlin: Springer-Verlag; 1983.

5. Kpikpi, JE, Thomas JD. A study of the sugar chemoreception niches of two bulinid snail hosts of schistosomiasis. Ann Trop Med Parasit. 1992;86:181-9.

6. Kumar P, Singh DK. Use of amino acids and their combinations as attractant in bait formulations against the snail Lymnaea acuminata. J Appl Biosci. 2009;35:63-6.

7. Kumar P, Singh DK. Molluscicidal activity of Ferula asafoetida, Syzygium aromaticum and Carum carvi and their active components against the snail Lymnaea acuminata. Chemosphere. 2006;63:1568-74

8. Kumar P, Singh DK. Amino acids and carbohydrates binary combination as an attractant in bait formulation against the snail Lymnaea acuminata. Malays Appl Biol. 2010;39:7-11.

9. Kumar P, Singh VK, Singh DK. Binary combination of amino acids and plant molluscicide in bait formulations against Lymnaea acuminata. World J Zool. 2010;5:143-7.
10. Kumar P, Singh VK, Singh DK. Kinetics of enzyme inhibition by active molluscicida agents ferulic acid, umbelliferone, eugenol and limonene in the nervous tissue of snail Lymnaea acuminata. Phytother Res. 2009;23:172-7.

11. Lowry, OH, Rosebrough NJ, Farr AL, Randall RJ Protein measurement with the Folin phenol reagents. J Biol Chem. 1951;193:265-75.

12. MacInnis AJ, Bethel WM, Cornford EM. Identification of chemicals of snail origin that attract Schistosoma mansoni miracidia. Nature.1974;248:361-3.

13. Madsen H. A comparative study on the food-locating ability of Helisoma duryi, Biomphalaria camerunensis and Bulinus truncatus (Pulmonata: planorbidae). J Appl Ecol. 1992;29:70-8.

14. Mas-Coma S, Valero MA, Bargues MD. Fasciola, Lymnaeids and human fascioliasis with a global overview on disease transmission, epidemiology, evolutionary genetics. molecular epidemiology and control. In: Rollinson D, Hay SI, editors. Advances in Parasitology, v. 69. Burlington: Academic Press; 2009. p. 41-146.

15. Schneider WC. Determination of nucleic acid in the tissue by pentose analysis In: Colowick SP, Kaplan NO, editors. Methods in enzymology, v. 14. New York: Academic Press; 1957. p. 680.

16. Singh P, Kumar P, Singh VK, Singh DK. Effect of snail attractant pellets containing plant molluscicides on certain enzymes in the nervous tissue of Lymnaea acuminata (Lamark). Bioscan. 2009;4:395-8.

17. Singh P, Singh DK. Binary combination of carbohydrates and amino acids as snai attractant in pellets containing molluscicides against the snail Lymnaea acuminata. Pestic Biochem Physiol. 2008;92:120-4.

18. Singh S, Singh VK, Singh DK. Effects of active molluscicidal agents of common spices on biochemical parameters in the ovotestis of Lymnaea acuminata. Malays Appl Biol. 1998;27:45-9.

19. Singh V, Singh DK. The effect of abiotic factors on the toxicity of cypermethrin against the snail Lymnaea acuminata in the control of fascioliasis. J Helminthol. 2009;83:39-45.

20. Sokal RR, Rohlf FJ. Introduction of biostatistics. San Francisco: W.H. Freeman 1973. p. $185-207$

21. Spice JR. Colorimetric procedure for amino acids. In: Colowick, SP; Kaplan NO, editors. Methods in Enzymology. New York: Academic Press; 1957. p. 464

22. Sterry PR, Thomas JD, Patience RL. Changes in the concentrations of short chain carboxylic acids and gases during decomposition of the aquatic macrophytes Lemna pacucicostata and Ceratophylum demersum. Freshwater Biol. 1985;15:139-53.

23. Thomas JD. Chemical ecology of the snail hosts of schistosomiasis; snail- snail and snail-plant interaction. Malacology. 1982;22:81-91.

24. Tiwari F, Singh DK. Toxicity of plant derived molluscicides in attractant food pellet against snail Lymnaea acuminata. Iranian J Pharmacol Ther. 2007;6:103-7.

25. Tiwari F, Singh DK. Attraction to amino acids by Lymnaea acuminata, snail host of Fasciola species. Braz J Med Biol Res. 2004;37:587-90.

26. Tiwari F, Singh DK. Behavioural responses of the snail Lymnaea acuminata to carbohydrates in snail-attractant pellets. Naturwissenschaften. 2004;91:378-80.

27. Uhazy LS, Tanaka RD, MacInnis AJ. Schistosoma mansoni: identification of chemicals that attract or trap its snail vector, Biomphalaria glabrata. Science. 1978;201:924-6.

Received: 28 April 2011

Accepted: 12 August 201 


\section{Revista do Instituto de Medicina Tropical de São Paulo on line.}

Publications from 1990 to the present data are now available on:

http://www.scielo.br/rimtsp

PAST ISSUES 1959-1989 (PDF)

www.imt.usp.br/portal/

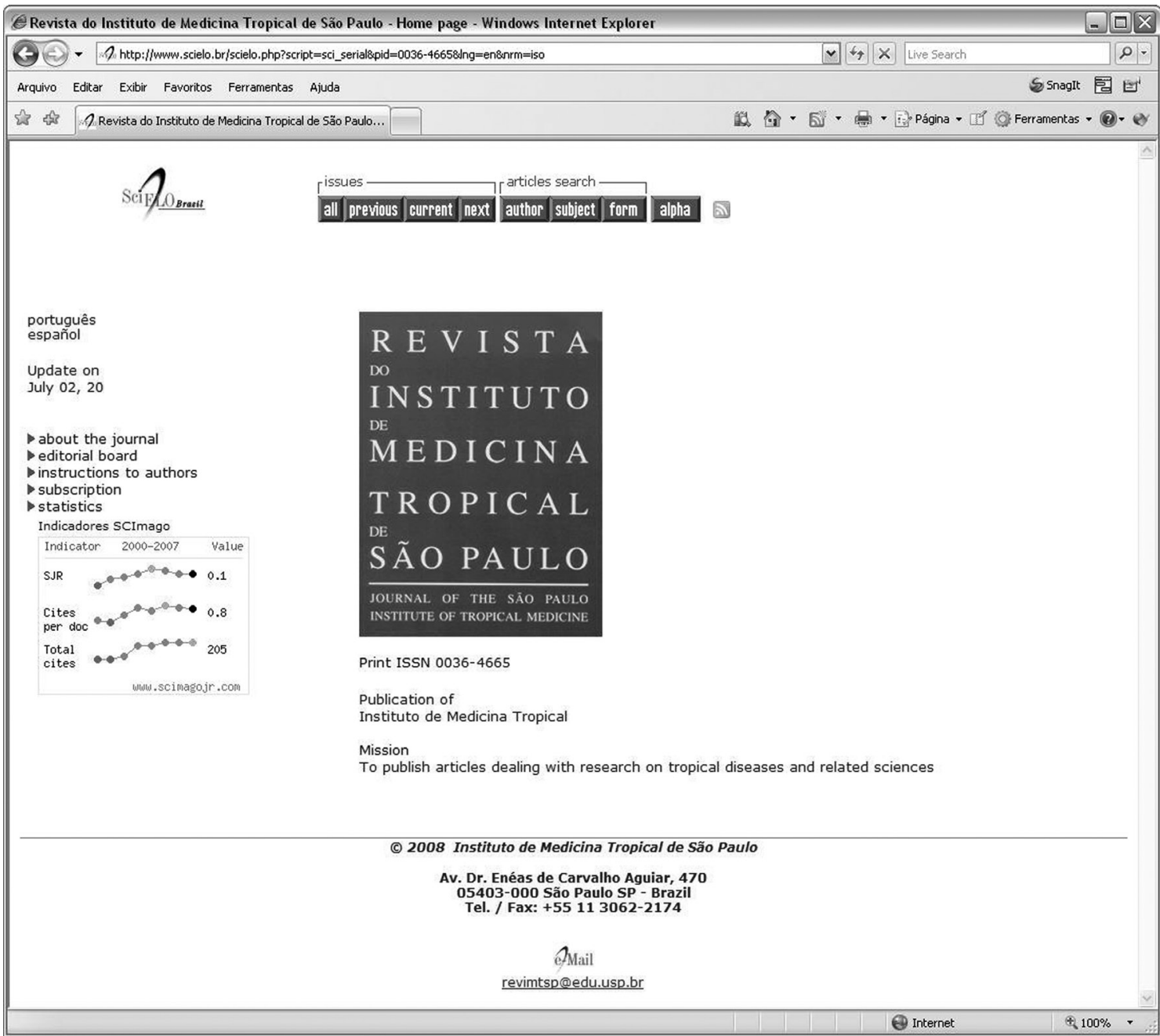

SciELO - The Scientific Electronic Library OnLine - SciELO is an electronic virtual covering a selected collection of Brazilian scientific journals.

The library is an integral part of a project being developed by FAPESP - Fundação de Amparo à Pesquisa do Estado de São Paulo, in partnership with BIREME - the Latin American and Caribbean Center on Health Sciences Information.

SciELO interface provides access to its serials collection via an alphabetic list of titles or a subject index or a search by word of serial titles, publisher names, city of publication and subject.

The interface also provides access to the full text of articles via author index or subject index or a search form on article elements such as author names, words from title, subject and words from full text.

FAPESP/BIREME Project on Scientific Electronic Publications Latin American and Caribbean Center on Health Sciences Information

Rua Botucatu 862 - 04023-901 São Paulo, SP - Brazil

Tel. (011) 5576-9863

scielo@bireme.br 